University of Nebraska - Lincoln

DigitalCommons@University of Nebraska - Lincoln

USDA Wildlife Services - Staff Publications

U.S. Department of Agriculture: Animal and Plant Health Inspection Service

2012

\title{
Detection of volatile organic compounds in cattle naturally infected with Mycobacterium bovis
}

\author{
Nir Peled \\ Tel-Aviv University, peled.nir@gmail.com \\ Radu lonescu \\ Israel Institute of Technology \\ Pauline Nol \\ US Department of Agriculture, pauline.nol@aphis.usda.gov \\ Orna Barash \\ Israel Institute of Technology \\ Matt McCollum \\ US Department of Agriculture
}

See next page for additional authors

Follow this and additional works at: https://digitalcommons.unl.edu/icwdm_usdanwrc

Peled, Nir; Ionescu, Radu; Nol, Pauline; Barash, Orna; McCollum, Matt; VerCauteren, Kurt C.; Koslow, Matthew; Stahl, Randal S.; Rhyan, Jack; and Haick, Hossam, "Detection of volatile organic compounds in cattle naturally infected with Mycobacterium bovis" (2012). USDA Wildlife Services - Staff Publications. 1178.

https://digitalcommons.unl.edu/icwdm_usdanwrc/1178

This Article is brought to you for free and open access by the U.S. Department of Agriculture: Animal and Plant Health Inspection Service at DigitalCommons@University of Nebraska - Lincoln. It has been accepted for inclusion in USDA Wildlife Services - Staff Publications by an authorized administrator of DigitalCommons@University of Nebraska - Lincoln. 


\section{Authors}

Nir Peled, Radu Ionescu, Pauline Nol, Orna Barash, Matt McCollum, Kurt C. VerCauteren, Matthew Koslow, Randal S. Stahl, Jack Rhyan, and Hossam Haick 


\title{
Detection of volatile organic compounds in cattle naturally infected with Mycobacterium bovis
}

\author{
Nir Peled ${ }^{\mathrm{a}, 1}$, Radu Ionescu ${ }^{\mathrm{b}, 1,2}$, Pauline Nol ${ }^{\mathrm{c}, 1}$, Orna Barash ${ }^{\mathrm{b}}$, Matt McCollum ${ }^{\mathrm{c}}$, Kurt VerCauteren ${ }^{\mathrm{c}}$ \\ Matthew Koslow ${ }^{\mathrm{a}}$, Randal Stahl ${ }^{\mathrm{c}}$, Jack Rhyan ${ }^{\mathrm{c}, *}$, Hossam Haick ${ }^{\mathrm{b}, * *}$ \\ a The Thoracic Cancer Research and Detection Center, Sheba Medical Center, Tel-Aviv University, Tel-Aviv 52621, Israel \\ ${ }^{\mathrm{b}}$ The Department of Chemical Engineering and Russell Berrie Nanotechnology Institute, Technion - Israel Institute of Technology, Haifa 32000, Israel \\ c The National Wildlife Research Center, US Department of Agriculture, Animal and Plant Health Inspection Service, Fort Collins, CO 80521, USA
}

\section{A R T I C L E I N F O}

\section{Article history:}

Received 21 March 2012

Received in revised form 30 April 2012

Accepted 8 May 2012

Available online 18 May 2012

\section{Keywords:}

Bovine tuberculosis

Mycobacterium bovis

Breath analysis

Volatile organic compound

Chemical sensors array

Gas-chromatography/mass-spectrometry

\begin{abstract}
A B S T R A C T
We report here on a novel methodology in detecting Mycobacterium bovis (M. bovis) infection in cattle, based on identifying unique volatile organic compounds (VOCs) or a VOC profile in the breath of cattle. The study was conducted on an M. bovis-infected dairy located in southern Colorado, USA, and on two tuberculosis-free dairies from northern Colorado examined as negative controls. Gaschromatography/mass-spectrometry analysis revealed the presence of 2 VOCs associated with $M$. bovis infection and 2 other VOCs associated with the healthy state in the exhaled breath of M. bovis-infected and not infected animals, yielding distinctly different VOC patterns for the two study groups. Based on these results, a nanotechnology-based array of sensors was then tailored for detection of $M$. bovis-infected cattle via breath. Our system successfully identified all $M$. bovis-infected animals, while $21 \%$ of the not infected animals were classified as $M$. bovis-infected. This technique could form the basis for a real-time cattle monitoring system that allows efficient and non-invasive screening for new $M$. bovis infections on dairy farms.
\end{abstract}

(c) 2012 Elsevier B.V. All rights reserved.

\section{Introduction}

Bovine tuberculosis (bTB), caused by Mycobacterium bovis (M. bovis), is a serious global disease with an impact on animal health, public health, and international trade [1-3]. The transmission of tuberculosis to humans via infected milk was considered a significant cause of morbidity and mortality from Victorian times until the Second World War [4,5]. Milk pasteurization and intensive eradication programs led to sharp declines of bTB in domestic livestock and humans, especially in developed countries [6]. However, the challenge of eradication remains, largely due to unauthorized

Abbreviations: bTB, bovine tuberculosis; M. bovis, Mycobacterium bovis; VOC, volatile organic compound; CFT, caudal fold test; CCT, comparative cervical test; GC-MS, gas chromatography-mass spectrometry; DFA, discriminant factor analysis; GNP, gold nanoparticles; TP, true positive; TN, true negative; FP, false positive; FN, false negative; $\mathrm{CV}$, canonical variable.

* Corresponding author at: The National Wildlife Research Center, US Department of Agriculture, Animal and Plant Health Inspection Service, Fort Collins, CO 80521 USA. Tel.: +1970266 6126; fax: +1970266 6138 .

** Corresponding author. Tel.: +972 4 8293087; fax: +972 778871880.

E-mail addresses: Jack.C.Rhyan@aphis.usda.gov (J. Rhyan)

hhossam@technion.ac.il (H. Haick).

1 These authors have contributed equally to the manuscript.

2 Present address: Department of Electronics, Electrical and Automatic Engineer-

ing, Rovira i Virgili University, 43007 Tarragona, Spain. movement of infected animals and persistent wildlife reservoirs of the disease $[7,8]$.

Accurate and efficient detection of bTB in animal populations remains of paramount importance to bTB control programs. Currently, tuberculosis testing in live cattle in the United States consists of a caudal fold test (CFT) as a screening test, with a comparative cervical test (CCT) or interferon gamma assay test as supplemental or confirmatory tests [3,9-11]. The CFT and CCT involve injecting tuberculin(s) intradermally and measuring any subsequent swelling at the site of injection $72 \mathrm{~h}$ later [12]. The interferon gamma assay is a confirmatory or supplemental blood test that relies on quantifying the amount of gamma interferon that is produced in animal blood samples cultured in the presence of tuberculin. The final diagnosis of bovine tuberculosis requires post mortem laboratory confirmation of disease via histopathology, polymerase chain reaction, and bacteriological culture $[9,10]$.

Although these combined tests have good specificity and sensitivity depending on the stage of infection (over $80 \%$ sensitivity and over $90 \%$ specificity, respectively), conducting these tests at large dairies is expensive, time-consuming, logistically challenging, and must be performed by certified veterinarians [13]. Skin testing requires a second examination, and the interferon gamma assay is considerably more expensive in comparison with a skin test $[14,15]$. Both interferon gamma and skin testing results are delayed a minimum of $48-72 \mathrm{~h}[13,15]$. The development of a 
sensitive, specific, non-invasive, and efficient method for detecting $M$. bovis infection, which can be performed on the premises, would be highly beneficial to both regulators and managers in the livestock industry.

An emerging approach for diagnosing, an infectious disease at its earliest stages relies on volatile organic compounds (VOCs) that are emitted from the infectious agent and/or the host. The successful analysis of infectious disease-related VOCs is based on the following principles of cell biology. The bacterial cell membrane consists primarily of amphipathic phospholipids, carbohydrates and many integral membrane proteins that are distinct for different cell types. In disease formation, both host and invading cells can undergo structural changes, one example of which would be oxidative stress, i.e., a peroxidation of the cell membrane that causes VOCs to be emitted [16]. Some of these VOCs appear in distinctively different mixture compositions [17-22]. What is particularly significant about this approach is that each type of disease has its own unique pattern of VOCs; therefore, the presence of one disease would not mask other disease types [23]. These VOCs can be detected directly from: (i) cultured cells (i.e., the mixture of VOCs trapped above the cells in a sealed vessel) [20-22]; (ii) urine [24]; or (iii) exhaled breath [17-19].

In regard to exhaled breath, the principle is that disease-related changes in blood chemistry are reflected in measurable changes to the breath through exchange via the lungs. In certain instances, breath testing offers several potential advantages, such as (a) breath samples are non-invasive and relatively easy to obtain, and (b) breath testing has the potential for direct, inexpensive and eventually real-time monitoring.

In this paper, we explore the utility of breath testing for the detection of $M$. bovis infection in cattle. We analyze breath samples collected from cattle using gas-chromatograph/mass-spectrometry (GC-MS) to identify the VOC patterns linked with the disease conditions. Based on the detected VOC patterns, a nanotechnology-based array of sensors, termed Nano Artificial NOSE (NA-NOSE) [25-30], was tailored for the detection of bTB disease from exhaled breath. NA-NOSE is an artificial olfactory system based on an array of crossreactive, nanomaterials-based, chemical gas sensors, which can identify and separate different gaseous mixtures, even if their constituent analytes are present at very low concentrations and their differences are very subtle. The results obtained indicate that the NA-NOSE could efficiently detect $M$. bovis infection from breath samples of cattle.

\section{Materials and methods}

\subsection{Breath collection from cattle}

Breath samples were collected and tested from 14 cattle from an $M$. bovis-infected dairy in the southern part of the state of Colorado, USA. Ten of these animals were identified as bTB-positive based on conventional tests. Nine of 10 cattle were culture positive for $M$. bovis at necropsy. One animal was culture negative but had gross and microscopic lesions compatible with bTB which were polymerase chain reaction positive. Nine of 9 animals tested were positive on CFT, 9 of 10 animals tested were positive on CCT, 8 of 10 animals tested on interferon gamma assay were positive and 2 were suspect; all 10 animals had gross lesions and 8 animals had microscopic lesions compatible with bTB. The remaining four animals from the same dairy were deemed bTB-negative based on the following: none of the animals had gross lesions, three of three animals tested were negative on CFT, one of one animal tested was negative on CCT, and one of one was negative on gamma interferon. Only one animal was cultured and it was negative, whereas the others were not cultured because they were negative on skin tests and

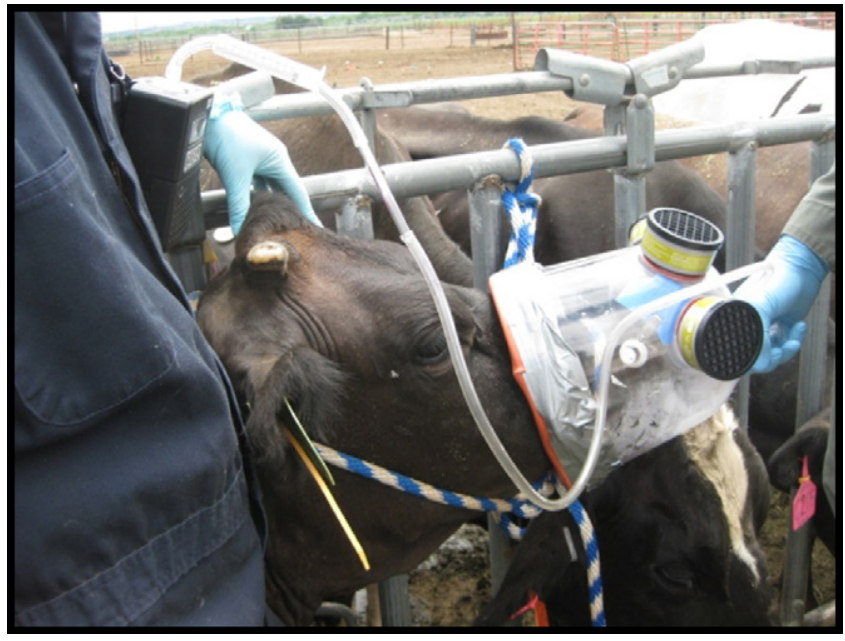

Fig. 1. Photo illustrating the system employed for breath sample collection in the cattle. Inspired air first passes into the mask through three charcoal filters and one-way valves to remove environmental VOCs. Expired air passes out of the mask through two one-way valves and through the tubing inserted into a hole in the front of the mask. Air in the tubing passes through a glass cartridge containing sorbent material (Tenax ${ }^{\mathrm{TM}}$ ) and is exhausted through the hand-held suction pump.

gamma interferon tests and they had no evidence of disease on post mortem examination. Additionally, breath samples from 13 cattle from two bTB-negative dairies located in northern Colorado were also tested. These animals served as negative controls, as well as to exclude confounders caused by farm and feed differences. These animals were not skin tested.

Breath specimens were collected by use of a mask designed to deliver nebulized medication to horses (Aeromask ${ }^{\circledR}$, Trudell Medical International, London, Ontario, Canada) modified so that inspired air passed through charcoal filter cartridges (North Safety Products by Honeywell, Cranston, RI, USA) and air in the mask was pumped via Tygon ${ }^{\circledR}$ tubing (Saint-Gobain Performance Plastics, Akron, $\mathrm{OH}, \mathrm{USA}$ ) through a glass cartridge containing inert sorbent material (Tenax ${ }^{\mathrm{TM}}$ Catalog No. 226-35-03, SKC Inc. Eighty Four, PA, USA) by means of a handheld pump (Air Check XR5000, SKC) (Fig. 1). This approach is necessary to reduce as much as possible any confounders or contaminants that occur external to the animals we are targeting. Air was sampled from the mask at a rate of $1 \mathrm{~L} / \mathrm{min}$ for $2 \mathrm{~min}$. The sorbent material concentrated the VOCs in the $2 \mathrm{~L}$ gas sample that passed through the tube. Following exposure, the sorbent tubes were sealed and stored at $-70^{\circ} \mathrm{C}$ until shipment to Israel for GC-MS and NA-NOSE analyses. The experiment was performed in compliance with the U.S. laws for the humane treatment of animals and was done in conjunction with disease management procedures of the Colorado Department of Agriculture and the U.S. Department of Agriculture, Animal and Plant Health Inspection Service, Veterinary Services.

\subsection{Breath analysis using the GC-MS}

The chemical composition of the breath samples collected from all 27 cattle was analyzed employing a Gas Chromatography-Mass Spectrometry equipment (GC-MS-QP2010; Shimadzu Corporation, Japan), combined with a thermal desorption system (TD20; Shimadzu Corporation, Japan). The GC oven temperature profile used was (i) $35^{\circ} \mathrm{C}$, hold for $10 \mathrm{~min}$; (ii) ramp of $4{ }^{\circ} \mathrm{C} / \mathrm{min}$ until $150^{\circ} \mathrm{C}$; (iii) ramp of $10^{\circ} \mathrm{C} / \mathrm{min}$ until $300^{\circ} \mathrm{C}$; and (iv) hold for $15 \mathrm{~min}$ at $300^{\circ} \mathrm{C}$. VOCs were chromatographically separated using an SLB$5 \mathrm{~ms}, 30 \mathrm{~m} \times 0.25 \mathrm{~mm}, 0.5 \mu \mathrm{m}$ film thickness, with $5 \%$ phenyl methyl siloxane, capillary column (Sigma Aldrich Ltd., Rehovot, Israel). The injection port was configured in a splitless injection mode 
at $23.4 \mathrm{kPa}$ for $2 \mathrm{~min}$, resulting in airflow with a constant linear velocity of $30.0 \mathrm{~cm} / \mathrm{s}$ and a column flow of $0.70 \mathrm{ml} / \mathrm{min}$. Samples in the sorbent tubes were desorbed at $250^{\circ} \mathrm{C}$. The molecular structures of the VOCs were determined by spectral library matching, using the Automated Mass Spectral Deconvolution and Identification System software. Ion profiles $(\mathrm{m} / \mathrm{z})$ were processed using the open source XCMS package version 1.22 .1 for $\mathrm{R}$ environment (http://metlin.scripps.edu/xcms/).

\subsection{Breath analysis using the NA-NOSE}

Twenty-two breath samples collected from the cattle (8 bTBpositive and 4 bTB-negative from the infected dairy, and 10 animals from the non-infected dairies) were analyzed with our specifically designed NA-NOSE system (Fig. 2a). Two sorbent tubes from animals confirmed as bTB-positive and three tubes from the tuberculosis-free dairies were damaged during shipment and could not be analyzed with the NA-NOSE. For the NA-NOSE, an array of six chemiresistive films of gold nanoparticle (GNP) sensors (GNP with either octadecanethiol (2 items), decanethiol, 2-naphthalenethiol, 2-mercaptobenzoazole or 2-nitro-4-trifluoromethylbenzenethiol) was selected from an initial pool of 18 sensors (Fig. 2b) based on the results of the GC-MS analysis, whereby the organic functionalities provided broadly cross-selective absorption sites for the breath VOCs [25-29,31,32]. The sensors were produced by successively drop casting the molecularly modified GNP solutions onto pre-prepared circular Ti/Au interdigitated electrodes (24 pairs of Au electrodes; $5 \mu \mathrm{m}$ width and $25 \mu \mathrm{m}$ spacing between the adjacent electrodes) on a silicon wafer with $1000 \mathrm{~nm} \mathrm{SiO}_{2}$ film and by wire-bonding the electrodes to TO5 package holder (National Semiconductor, US) - see Fig. 2c. In these sensing films (Fig. 2d), the gold particles provide the electric conductivity and the organic film component provides sites for the sorption of analyte (guest) molecules. Details of the sensing materials synthesis have been described elsewhere [25-29,31,32].

For analyzing the breath samples with the NA-NOSE, the breath samples collected were introduced into a $400 \mathrm{~mL}$ sealed test chamber, housing the sensors (see Fig. 2a). Samples were thermally desorbed at $270^{\circ} \mathrm{C}$ from the Tenax ${ }^{\mathrm{TM}}$ cartridge using a $750 \mathrm{~mL}$ gas stream into the sample chamber. Sensors output was monitored for a change in resistance using a custom program (LabView, National Instruments). All sensors were monitored simultaneously through an Agilent 34980A multifunction switch. A Stanford Research System SR830 DSP lock-in amplifier controlled by an IEEE 488 bus was used to supply the AC voltage signal to the sensors $(0.2 \mathrm{~V}$ at $1 \mathrm{kHz})$, and to measure the corresponding current $(<10 \mu \mathrm{A}$ in the studied devices). This setup allows for measuring normalized changes in conductance as small as $0.01 \%$. The sensors system was degassed under vacuum for $5 \mathrm{~min}$ at a pressure of $<50$ mtorr, prior to analyzing another sample, in order to purge the test chamber.

\subsection{Data analysis}

The GC-MS results for a given identified compound were compared across three treatment groups using the Wilcoxon rank sum test at a significance level of $p$-value $<0.05$ [33]: bTB positive animals from the infected dairy, bTB negative animals from the infected dairy, and animals from the tuberculosis-free dairies. NA-NOSE sensors responses, defined as the relative resistance change experienced by the sensors immediately after exposure to the breath sample, were used as inputs for Discriminant Factor Analysis (DFA) pattern recognition algorithm [34,35]. Data were selected to be used as training and validation data sets. The classification prediction was calculated employing the leave-one-out cross-validation method, as described elsewhere [36]. For this purpose, DFA was computed using a training data set that excluded one (a)

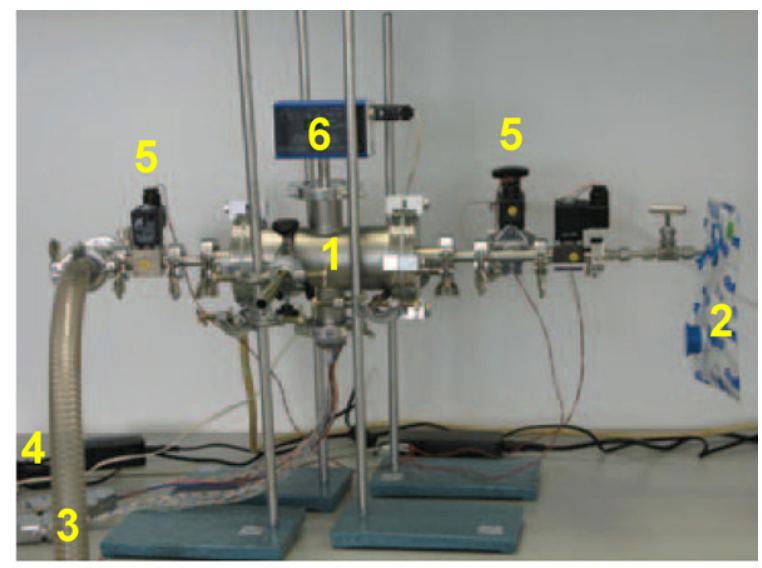

(b)

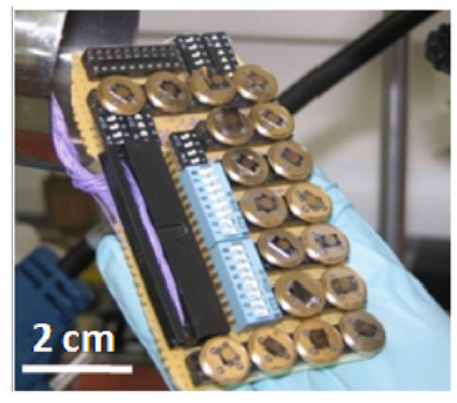

(c)

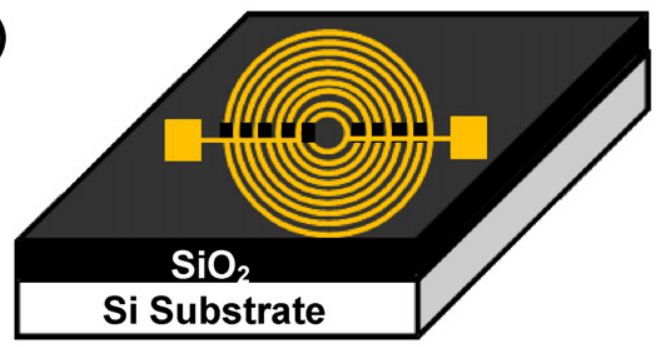

(d)

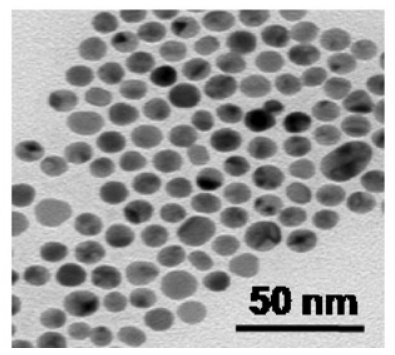

Fig. 2. (a) Photo of the NA-NOSE measurement system: 1 - sensors test chamber, 2 - breath sample bag, 3 - tubing to the vacuum pump, 4 - data acquisition board, 5 - automatically controlled valves, 6 - vacuum meter; (b) photo of the array of chemisensors; (c) schematic representation of sensors substrate (not drawn to scale); (d) tunneling electron micrograph image of the GNP sensing film.

test sample. After the DFA computation, the test sample was projected onto the DFA model that was calculated using the training set. In this way, the test sample was blind for the DFA model, so that its class affiliation was unknown. In a two-group classification case, the discrimination is obtained through the first canonical variable (CV1). The classification of the unknow sample was determined using standard cluster analysis based on the distance to groups centers on CV1-axis. All possibilities of leaving out one sample were tested, and the left-out sample was classified as true positive (TP), true negative (TN), false positive (FP) and false negative (FN). bTB 


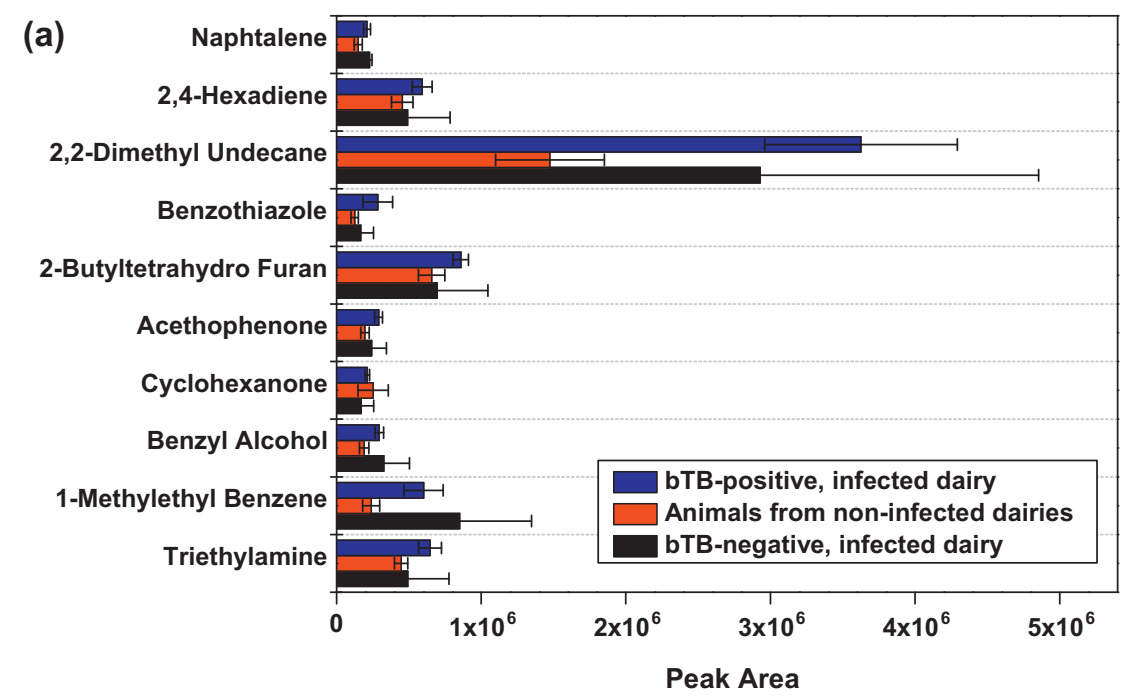

(b)

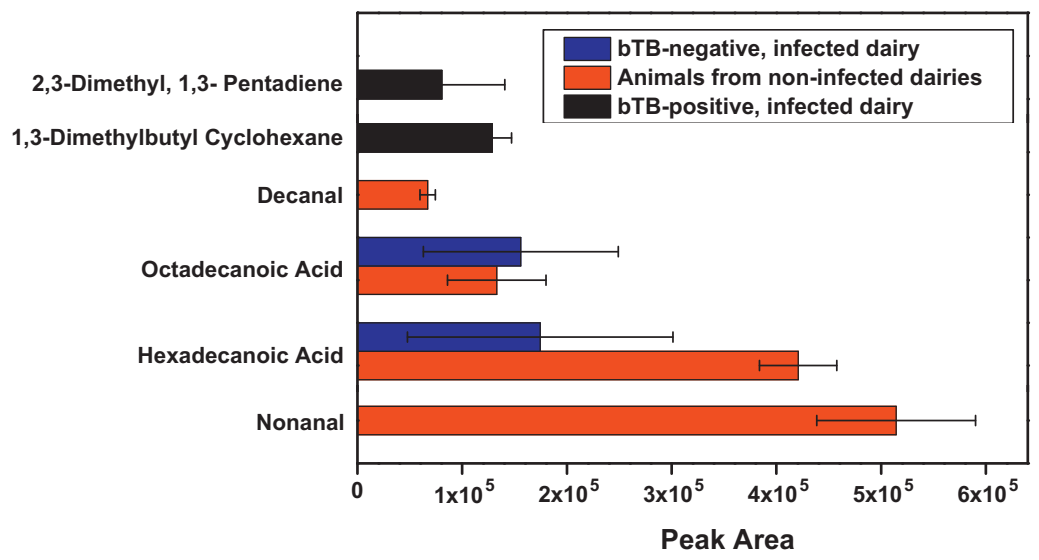

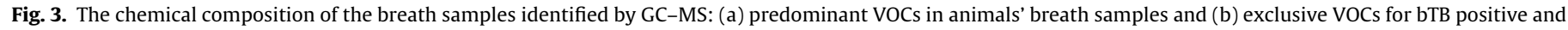
bTB negative cattle.

identification sensitivity and specificity were calculated from Eq. (1):

Sensitivity $=\frac{\mathrm{TP}}{\mathrm{TP}+\mathrm{FN}} ; \quad$ Specificity $=\frac{\mathrm{TN}}{\mathrm{TP}+\mathrm{FP}}$

Features extraction and data classification were conducted under the MATLAB ${ }^{\circledR}$ (The MathWorks) environment. Statistical analysis was carried out using SAS JMP, Version 8.0 (SAS Institute Inc., Cary, NC, USA, 1989-2005).

\section{Results}

\subsection{Chemical analysis of the breath samples}

The chemical composition of the breath samples analyzed by GC-MS included the following types of compounds: two ketones, two aromatic compounds, one methylated alkane, one cycloalkane, one ether compound, one alcohol, one benzene derivative, one amine, two dienes, two aldehydes, and two acids. All but six compounds were found in the exhaled breath of the majority of cattle, being present in at least $80 \%$ of bTB positive cattle's breath samples and in at least 75\% of bTB negative cattle's breath samples (Fig. 3a). These compounds cannot be associated with bTB infection since the relative concentrations of the 10 analytes common to both groups, based on differences in peak areas for an identified compound, were not statistically similar for bTB negative animals independent of their dairy of origin, whereas statistically different from the bTB infected animals, as resulting from the Wilcoxon tests performed. The remaining six compounds were further investigated due to their presence in the breath of more than $75 \%$ of the specimens from one group and in less than $25 \%$ of the specimens from the other group. These compounds were: one cycloalkane and one diene for bTB, and two aldehydes and two acids for not infected animals (Fig. 3b). As the two aldehydes were found only in the breath of the animals from the tuberculosis-free dairies, and not in the breath of the bTB negative animals from the infected dairy, they were excluded from the development of the GNP sensors array.

\subsection{Analysis of breath samples with NA-NOSE}

The NA-NOSE ultimately comprised an array of six crossreactive GNP sensors which were selected based on their ability to discriminate the VOC patterns identified by the GC-MS. The six chemiresistive films of GNP were coated with either octadecanethiol (2 sensors), decanethiol, 2-naphthalenethiol, 2mercaptobenzoazole or 2-nitro-4-trifluoro-methylbenzenethiol. Although employing the same active material, the two sensors coated with octadecanethiol were not identical because they presented different baseline resistance values. Each of the six GNP sensors of the reservoir responded either to all or to a certain subset of the VOCs found in the samples, because the organic ligands of the GNPs provided only a moderate chemical selectivity. Each 


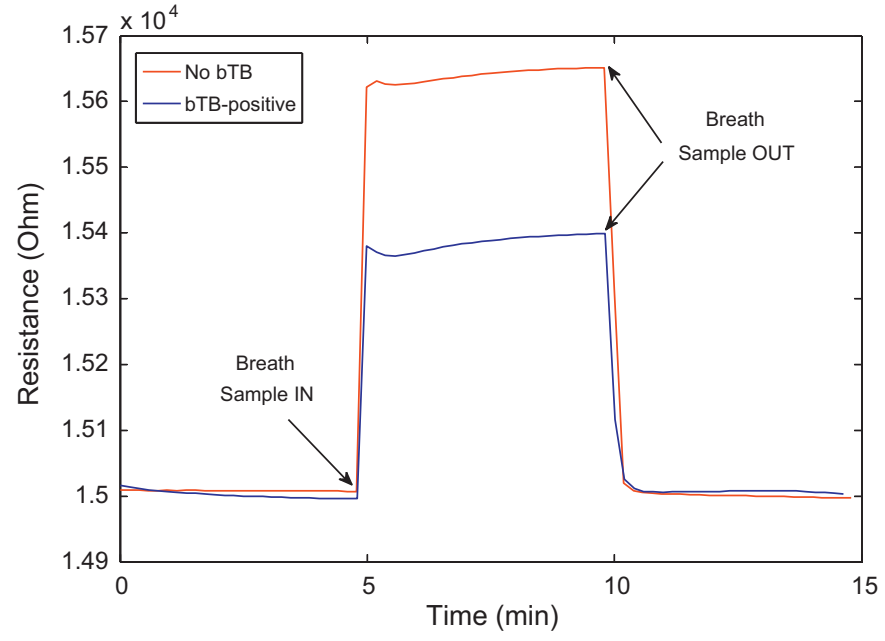

Fig. 4. Response of the GNP coated with octadecanethiol sensor to the breath samples of one animal from a tuberculosis-free dairy (red curve) and one bTB-positive animal from the $M$. bovis-infected dairy (blue curve). (For interpretation of the references to color in this figure legend, the reader is referred to the web version of the article.)

sensor from the array underwent a rapid and fully reversible change (increase) in electrical resistance upon exposure to the samples. Fig. 4 shows a typical response of one of the sensors, whose resistance increased as a consequence of a swelling effect produced when its active (sensing) material trapped the VOCs from the breath samples [37,38]. This figure suggests a higher affinity of the sensor to the VOC pattern correlated to an uninfected animal.

DFA analysis was then performed with the aim of identifying the bTB-infected cattle from the other animals based on breath analysis. The DFA plot obtained employing the features extracted from sensors responses is shown in Fig. 4. Using a blind leave-one-out cross-validation procedure, the NA-NOSE system correctly identified all bTB positive animals, while three out of the $14 \mathrm{bTB}$ negative cows were misclassified; one from the infected dairy and two from the non-infected dairies. The contingency table of samples classification is shown in Table 1 . Overall, based on our small study of 22 animals, the sensitivity and specificity were $100 \%$ and $79 \%$, respectively.

\section{Discussion}

So far, GC-MS is beneficial for detection of VOCs that are above the instrument's limit of detection. For cases where the GC-MS can detect and identify bTB breath VOCs, several factors impede its practical implementation in point-of-care or end-user sites. These limitations include the need for expensive equipment, the high levels of expertise required to operate such instruments, the speed required for sampling and analysis, and the need for preconcentration techniques. For bTB breath analysis to become a reality, we have utilized the GC-MS results obtained in the current study and designed a tailor-made NA-NOSE that is small, easy-to-use, inexpensive, and can detect VOCs in the presence of water vapor without the need for preconcentration and/or dehumidification techniques.

Table 1

Contingency table of bTB identification obtained by the NA-NOSE system.

\begin{tabular}{llll}
\hline & \multicolumn{3}{l}{ Actual value } \\
\cline { 3 - 4 } & & Positive & Negative \\
\hline Prediction outcome & Positive & $\mathrm{TP}=8$ & $\mathrm{FP}=3$ \\
& Negative & $\mathrm{FN}=0$ & $\mathrm{TN}=11$ \\
\hline
\end{tabular}

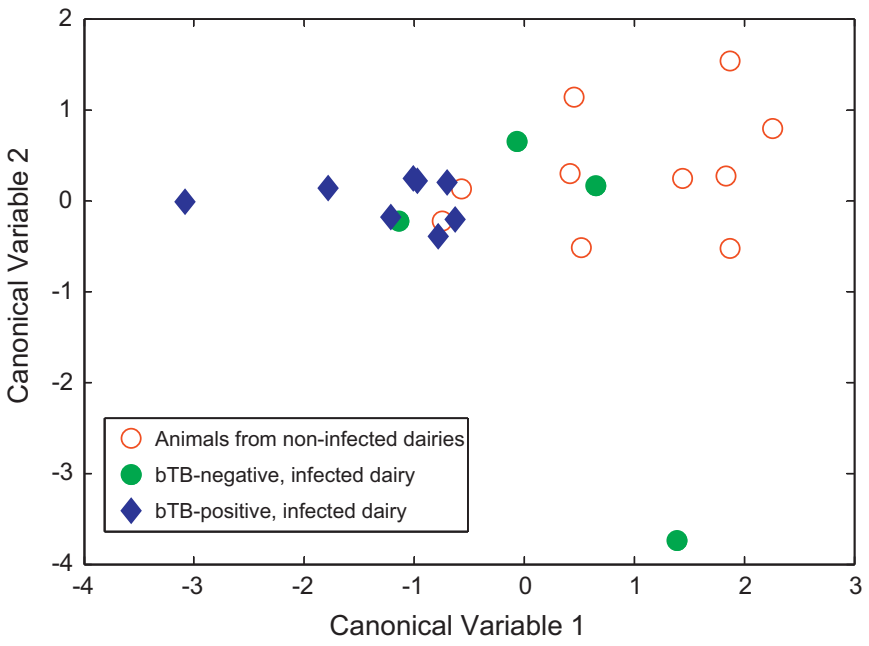

Fig. 5. DFA plot showing the discrimination between bTB-negative (circles; $n=14$ ) and bTB-positive (diamonds; $n=8$ ) cattle. An insight of the bTB-negative animals from the different dairies shows the complete mixing of the animals from $M$. bovis infected (green filled circles; $n=4$ ) and tuberculosis-free (red open circles; $n=10$ ) dairies. (For interpretation of the references to color in this figure legend, the reader is referred to the web version of the article.)

With our NA-NOSE system, we were able to correctly identify cattle naturally infected with $M$. bovis and $79 \%$ of bTB negative animals in this study. This is the first known report of application of VOC analysis to detect $M$. bovis in cattle breath. Previous studies in cattle have demonstrated different profiles of VOCs in the air over serum samples (headspace) from Brucella-infected, MAP-infected, M. bovis-infected, and normal cattle using an e-nose $[39,40]$. Studies in humans to detect tuberculosis in breath VOCs have been performed with some success [41-44].

It remains unknown why the three negative animals were misclassified as positive. Since the negative animals selected from the southern Colorado dairy did not undergo complete workups such as the positive animals did, it is possible that these cattle were exposed to $M$. bovis and were in very early stages on infection. There is also the possibility that these cattle, especially the animals from the bTB-free dairies, are undergoing an infection process that produces volatiles that "cross-react" in the NA-NOSE system used in this study. It is important to note that the bTB negative animals were independent of their dairy of origin, this affirmation being supported by the complete mixing of the bTB negative animals (Fig. 5), which indicates that the NA-NOSE was not affected by confounding factors determined by the dairy of origin. Our results suggest that the trained NA-NOSE system is responsive to a volatile biomarkers pattern related to $M$. bovis infection.

Importantly, the cyclohexane and the pentadiene identified as exclusive VOCs for $M$. bovis infection could not be found as prevalent in the breath of the bTB negative animals from the infected dairy, therefore they are not dairy dependent and could indeed represent tentative biomarkers for $M$. bovis identification. The two saturated fatty acids not found in the breath of the bTB infected animals were found in the breath of all bTB negative animals, also independent of their dairy location, which indicates that they were reduced during disease growth, which is in agreement with previous findings that fatty acids can be taken up by $M$. tuberculosis from the triton-fatty acid complex and utilized as a source of carbon for growth [45]. The two aldehydes that were excluded from the NANOSE sensor array are probably associated with feed differences among the different dairies. Most of the VOCs found in this study are similar in structure to the compounds found in previous studies done by Phillips et al. both in humans and in vitro [41,42]. However, there were some compounds found by Phillips et al. which were not 
found in this study (i.e., more numerous alkane derivatives, alkene and ester), while no alcohols, amine and aromatic compounds were found in their studies. This difference may be due to the fact that previous studies were conducted on humans and on in vitro cultures and this study was in cattle.

Identification of the sensors that contributed most to differentiating bTB positives from negatives was crucial for identifying the bTB patterns. GNP sensors developed by Haick and coworkers have been shown to be sensitive to typical breath VOCs such as aldehydes, alkanes, ketones, alcohols, and benzene derivatives, with typical detection limits for the separate VOCs of 1-5 parts per billion (ppb), and showed a very low response to water [27], an important feature because the high background humidity in breath samples could easily mask the signal to the much lower concentrations of the VOCs that indicate bTB state. Breath specimens from the bTB positive and bTB negative animals were characterized by subtle differences in the concentration of a multitude of metabolites. On the other hand, the concentrations of many other metabolites remained unaffected. Some of the GNP sensors were especially sensitive to the classes of bTB-infected specific VOCs. Nevertheless, the majority of the sensors were more sensitive to the VOCs that were unaffected by the bTB state, and mainly added noise, therefore they were discarded.

\section{Conclusions}

We report on a new methodology in detecting $M$. bovis infection in cattle, based on identifying unique VOCs or a VOC profile in the breath of cattle. GC-MS analysis revealed the presence of two VOCs associated with $M$. bovis infection and two other VOCs associated with the healthy state in the exhaled breath of M. bovisinfected and not infected animals, yielding distinctly different VOC patterns for the two study groups. Based on these results, a custommade nanotechnology-based array of sensors (NA-NOSE) was then tailored for detection of $M$. bovis-infected cattle via breath. Our system successfully identified all M. bovis-infected animals, while $21 \%$ of the not infected animals were classified as $M$. bovis-infected.

The NA-NOSE system we present here shows great promise as a screening technique for bovine tuberculosis in animal populations. The NA-NOSE has advantages over the GC-MS technique because it is faster, cheaper, and portable. It could be placed beside the milking line or at the barn entrance as a screening tool for bTB in cattle. This method might also hold potential as a screening test for other diseases, such as bovine brucellosis and paratuberculosis. Further studies on experimentally and naturally infected populations, spread over a larger geographical area, are necessary to fully verify and/or refine the observed breath biomarker patterns for bTB diagnosis.

\section{Acknowledgments}

We acknowledge Tamer Karawany and Moris Artoul (Technion - IIT) for technical assistance with breath analysis and Dr. Ulrike (Mirjam) Tisch (Technion - IIT) for fruitful discussions. We would also like to thank Tolani Francisco (USDA/Veterinary Services) and Robert Meyer (Wyoming State Livestock Board; Formerly USDA/Veterinary Services) for their technical assistance in the field.

\section{References}

[1] P.A. LoBue, D.A. Enarson, C.O. Thoen, Tuberculosis in humans and animals: an overview, International Journal of Tuberculosis and Lung Disease 14 (2010) 1075-1078.

[2] I. Schiller, B. Oesch, H.M. Vordermeier, M.V. Palmer, B.N. Harris, K.A. Orloski, B.M. Buddle, T.C. Thacker, K.P. Lyashchenko, W.R. Waters, Bovine tuberculosis: a review of current and emerging diagnostic techniques in view of their relevance for disease control and eradication, Transboundary and Emerging Diseases 57 (2010) 205-220.

[3] M.V. Palmer, W.R. Waters, Advances in bovine tuberculosis diagnosis and pathogenesis: what policy makers need to know, Veterinary Microbiology 112 (2006) 181-190.

[4] C.H. Collins, J.M. Grange, The bovine tubercle bacillus, Journal of Applied Microbiology 55 (1983) 13-29.

[5] C. Thoen, P. LoBue, I. de Kantor, The importance of Mycobacterium bovis as a zoonosis, Veterinary Microbiology 112 (2006) 339-345.

[6] R. De La Rua-Domenech, Human Mycobacterium bovis infection in the United Kingdom: incidence, risks, control measures and review of the zoonotic aspects of bovine tuberculosis, Tuberculosis 86 (2006) 77-109.

[7] M.F. Humblet, M.L. Boschiroli, C. Saegerman, Classification of worldwide bovine tuberculosis risk factors in cattle: a stratified approach, Veterinary Research 40 (2009).

[8] A.L. Michel, B. Müller, P.D. van Helden, Mycobacterium bovis at the animalhuman interface: a problem or not? Veterinary Microbiology 140 (2010) 371-381.

[9] L. Richeldi, Rapid identification of Mycobacterium tuberculosis infection, Clinical Microbiology and Infection 12 (2006) 34-36.

[10] N.W. Lerche, J.L. Yee, S.V. Capuano, J.L. Flynn, New approaches to tuberculosis surveillance in nonhuman primates, ILAR Journal 49 (2008) 170-178.

[11] California Department of Food \& Agriculture, TB Testing Information for Cattle Producers, http://www.cdfa.ca.gov/ahfss/animal_health/ pdfs/TB/TB_Testing_FS_081508.pdf (August 2008).

[12] M.V. Palmer, W.R. Waters, T.C. Thacker, R. Greenwald, J. Esfandiari, K.P. Lyashchenko, Effects of different tuberculin skin-testing regimens on gamma interferon and antibody responses in cattle experimentally infected with Mycobacterium bovis, Clinical and Vaccine Immunology 13 (2006) 387-394.

[13] A. Nienhaus, A. Schablon, J. Torres Costa, R. Diel, Systematic review of cost and cost-effectiveness of different TB-screening strategies, BMC Health Services Research 11 (2011) 1-10.

[14] S. Katsenos, M. Nikolopoulou, G. Tsiouri, I.D. Bassukas, S.H. Constantopoulos, The challenging evaluation of patients with severe psoriasis for latent tuberculosis: an important indication for IGRA, The Open Respiratory Medicine Journal 5 (2011) 59-60.

[15] I. Schiller, H.M. Vordermeier, W.R. Waters, A.O. Whelan, M. Coad, E. Gormley, B.M. Buddle, M. Palmer, T. Thacker, J. McNair, M. Welsh, R.G. Hewinson, B. Oesch, Bovine tuberculosis: effect of the tuberculin skin test on in vitro interferon gamma responses, Veterinary Immunology and Immunopathology 136 (2010) 1-11.

[16] C.M.F. Kneepkens, G. Lepage, C.C. Roy, The potential of the hydrocarbon breath test as a measure of lipid peroxidation, Free Radical Biology and Medicine 17 (1994) 127-160.

[17] J.I. Baumbach, Ion mobility spectroscopy. Developments for breath diagnostics and personalized medicine, GIT Labor-Fachzeitschrift 54 (2010) 198-201.

[18] S.T. Chambers, S. Bhandari, A. Scott-Thomas, M. Syhre, Novel diagnostics: progress toward a breath test for invasive Aspergillus fumigatus, Medical Mycology 49 (2011) S54-S61.

[19] M. Lechner, J. Rieder, Mass spectrometric profiling of low-molecular-weight volatile compounds - diagnostic potential and latest applications, Current Medicinal Chemistry 14 (2007) 987-995.

[20] J. Zhu, H.D. Bean, Y.-M. Kuo, J.E. Hill, Fast detection of volatile organic compounds from bacterial cultures by secondary electrospray ionization-mass spectrometry, Journal of Clinical Microbiology 48 (2010) 4426-4431.

[21] K. Naraghi, N. Sahgal, B. Adriaans, H. Barr, N. Magan, Use of volatile fingerprints for rapid screening of antifungal agents for efficacy against dermatophyte Trichophyton species, Sensors and Actuators B 46 (2010) 521-526.

[22] E. Barbieri, A.M. Gioacchini, A. Zambonelli, L. Bertini, V. Stocchi, Determination of microbial volatile organic compounds from Staphylococcus pasteuri against Tuber borchii using solid-phase microextraction and gas chromatography/ion trap mass spectrometry, Rapid Communications in Mass Spectrometry 19 (2006) 3411-3415.

[23] J. Dummer, M. Storer, M. Swanney, M. McEwan, A. Scott-Thomas, S. Bhandari, S. Chambers, R. Dweik, M. Epton, Analysis of biogenic volatile organic compounds in human health and disease, Trends in Analytical Chemistry 30 (2011) 960-967.

[24] K.M. Banday, K.K. Pasikanti, E.C.Y. Chan, R. Singla, K.V.S. Rao, V.S. Chauhan, R.K Nanda, Use of urine volatile organic compounds to discriminate tuberculosis patients from healthy subjects, Analytical Chemistry 83 (2011) 5526-5534.

[25] M. Hakim, S. Billan, U. Tisch, G. Peng, I. Dvrokind, R. Abdah-Bortnyak, A. Kuten, H. Haick, Diagnosis of head-and-neck-cancer from exhaled breath, British Journal of Cancer 104 (2011) 1649-1655

[26] G. Peng, M. Hakim, Y.Y. Broza, S. Billan, R. Abdah-Bortnyak, A. Kuten, U. Tisch, H Haick, Detection of lung, breast, colorectal, and prostate cancers from exhaled breath using a single array of nanosensors, British Journal of Cancer 103 (2010) 542-551.

[27] G. Peng, U. Tisch, O. Adams, M. Hakim, N. Shehada, Y.Y. Broza, S. Billan, R. AbdahBortnyak, A. Kuten, H. Haick, Diagnosing lung cancer in exhaled breath using gold nanoparticles, Nature Nanotechnology 4 (2009) 669-673.

[28] U. Tisch, H. Haick, Nanomaterials for cross-reactive sensor arrays, MRS Bulletin 35 (2010) 797-803.

[29] U. Tisch, H. Haick, Arrays of chemisensitive monolayer-capped metallic nanoparticles for diagnostic breath testing, Reviews in Chemical Engineering 26 (2011) 171-179. 
[30] R. Ionescu, Y. Broza, H. Shaltieli, D. Sadeh, Y, Zilberman, X. Feng, L. GlassMarmor, I. Lejbkowicz, K. Müllen, A. Miller, H. Haick, Detection of multiple sclerosis from exhaled breath using bilayers of polycyclic aromatic hydrocarbons and single-wall carbon nanotubes, ACS Chemical Neuroscience 2 (2011) 687-693.

[31] E. Dovgolevsky, G. Konvalina, U. Tisch, H. Haick, Monolayer-capped cubic platinum nanoparticles for sensing nonpolar analytes in highly humid atmospheres, Journal of Physical Chemistry C 114 (2010) 14042-14049.

[32] E. Dovgolevsky, U. Tisch, H. Haick, Chemically sensitive resistors based on monolayer-capped cubic nanoparticles: towards configurable nanoporous sensors, Small 5 (2009) 1158-1161.

[33] J.H. McDonald, Handbook of Biological Statistics, Sparky House Publishing, Baltimore, MD, USA, 2009

[34] R.G. Brereton, Chemometrics application of mathematics statistics to laboratory systems, Ellis Horwood, Chichester, 1990.

[35] R. Ionescu, E. Llobet, X. Vilanova, J. Brezmes, J.E. Sueiras, J. Caldererc, X. Correig, Quantitative analysis of $\mathrm{NO}_{2}$ in the presence of $\mathrm{CO}$ using a single tungsten oxide semiconductor sensor and dynamic signal processing, Analyst 127 (2002) 1237-1246.

[36] Y. Zilberman, R. Ionescu, X. Feng, K. Mullen, H. Haick, An array of polycyclic aromatic hydrocarbons and carbon nanotubes for accurate and predictive detection of organic compounds in real-world environmental humidity, ACS Nano 5 (2011) 6743-6753.

[37] Y.Joseph, B. Guse, T. Vossmeyer, A. Yasuda, Gold nanoparticle/organic networks as chemiresistor coatings: the effect of film morphology on vapor sensitivity, Journal of Physical Chemistry C 112 (2008) 12507-12514.

[38] F.J. Ibañez, F.P. Zamborini, Chemiresistive sensing of volatile organic compounds with films of surfactant-stabilized gold and gold-silver alloy nanoparticles, ACS Nano 2 (2008) 1543-1552.

[39] R. Fend, R. Geddes, S. Lesellier, H.M. Vordermeier, L.A.L. Corner, E. Gormley, E. Costello, R.G. Hewinson, D.J. Marlin, A.C. Woodman, M.A. Chambers, Use of an electronic nose to diagnose Mycobacterium bovis infection in badgers and cattle, Journal of Clinical Microbiology 43 (2005) 1745-1751.

[40] J. Knobloch, Volatile organic compound (VOC) analysis for disease detection: proof of principle for field studies detecting paratuberculosis and brucellosis, in: M. Pardo, G. Sberveglieri (Eds.), Olfaction and Electronic Nose, Proceedings, 2009.

[41] M. Phillips, R.N. Cataneo, R. Condos, G.A. Ring Erickson, J. Greenberg, V. La Bombardi, M.I. Munawar, O. Tietje, Volatile biomarkers of pulmonary tuberculosis in the breath, Tuberculosis (Edinburgh) 87 (2007) 44-52.

[42] M. Phillips, V. Basa-Dalay, G. Bothamley, R.N. Cataneo, P.K. Lam, M.P. Natividad, P. Schmitt, J. Wai, Breath biomarkers of active pulmonary tuberculosis, Tuberculosis (Edinburgh) 90 (2010) 145-151.

[43] M. Syhre, S.T. Chambers, The scent of Mycobacterium tuberculosis, Tuberculosis (Edinburgh) 88 (2008) 317-323.

[44] M. Syhre, L. Manning, S. Phuanukoonnon, P. Harino, S.T. Chambers, The scent of Mycobacterium tuberculosis. Part II: Breath, Tuberculosis 89 (2009) 263-266.

[45] L.W. Hedgecock, Complexing of fatty acids by triton WR1339 in relation to growth of Mycobacterium tuberculosis, Journal of Bacteriology 103 (1970) $520-522$.

\section{Biographies}

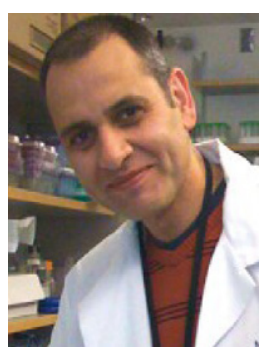

Nir Peled is a Pulmonologist and Thoracic Medical Oncologist. Dr. Peled is the head of the Research and Detection Unit for Thoracic malignancies at the Sheba Medical Center, Tel Hashomer, Israel and a Faculty member at the Sackler Faculty of Medicine, Tel Aviv University, Israel. Dr. Peled focuses on the wide clinical perspective of respiratory diseases and lung cancer and studies specifically the biomarkers of lung cancer for the early detection of this disease.

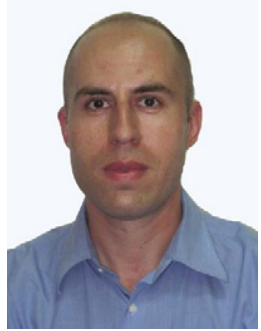

Radu Ionescu is an EC Senior Researcher in the Department of Chemical Engineering from the Technion - Israel Institute of Technology. He received his degree in Electrical Power Engineering from the Polytechnic University of Bucharest, Romania, in 1998, and his Ph.D. degree in Electronics Engineering from the Polytechnic University of Catalonia, Barcelona, Spain, in 2003. He was postdoctoral researcher at Rovira i Virgili University, Tarragona, Spain (4 years) and experienced researcher (FP6 Marie Curie Tranfer of Knowledge Actions) at Mediterranean University of Reggio Calabria, Italy (2 years).
Pauline Nol is a Veterinary Epidemiologist and Scientist with the Wildlife/Livestock Disease Investigations Team (WiLDIT) under the United States Department of Agriculture, Animal and Plant Health Inspection Service, Veterinary Services, Western Regional Office. WiLDIT is located at the National Wildlife Research Center in Fort Collins, CO, USA. Dr. Nol's research interests include the investigation, detection, and management of diseases that occur at the wildlife/livestock/human interface, with a special focus on wildlife vaccination.

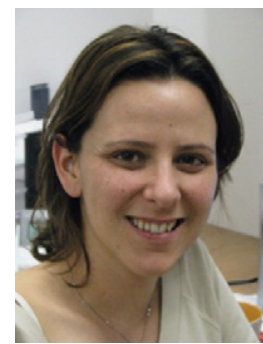

Orna Barash obtained her B.Sc. in Biochemical Engineering from the Technion - Israel Institute of Technology on 2008. Orna has joined Prof. Haick's group in 2007 during her final B.Sc. research project. Currently, Orna is a Ph.D. student in the Department of Chemical Engineering under the supervision of Prof. Hossam Haick. Her research is in identifying the specific "smell print" emitted in the headspace of in vitro lung cancer cell lines with different genetic mutations by means of nanoarrays and spectrometry techniques.

Matt McCollum is a Wildlife Biologist and a member of the Wildlife/livestock Disease Investigations Team located at the USDA, National Wildlife Research Cente in Fort Collins, CO, USA. Mr. McCollum's research interests include the epidemiology, detection, and management of infectious diseases at the wildlife/domestic animal/human interface, specifically, brucellosis and tuberculosis.

Kurt VerCauteren leads the "Management of Ungulate Disease and Damage" and "Rabies Management" projects at the National Wildlife Research Center of the United States Department of Agriculture/Animal Plant and Health Inspection Service/Wildlife Services. Dr. VerCauteren also chairs the Wildlife Damage Management Working Group of The Wildlife Society. His research focuses on human-wildlife conflict relative to wildlife damage management and diseases of wildlife that impact humans and livestock. Specifically, his current efforts focus on rabies in terrestrial wildlife, chronic wasting disease and bovine tuberculosis in deer and elk, and methods to reduce the multitude of damage caused by overabundant deer and elk

Matthew Koslow is a Specialist in Internal Medicine and senior fellow in Pulmonary Medicine at the Sheba Medical Center, Tel Hashomer, Israel. He received his Medica Degree from George Washington University School of Medicine, Washington, DC, USA (2002), Internal Medicine Residency, Albert Einstein College of Medicine, NY, USA (2005), Board Certified by American Board of Internal Medicine (2005) and Israeli Board of Internal Medicine (2009).

Randal Stahl is a Method Development Chemist at the USDA/APHIS/Wildlife Services/National Wildlife Research Center in Fort Collins, CO, USA. In addition to working on non-invasive methods for detecting disease in animal populations $\mathrm{Dr}$. Stahl works on methods to identify biomarkers of significance in managing wildlife populations.

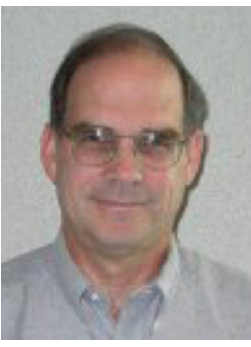

Jack Rhyan is a Veterinary Pathologist and the Leader of the Wildlife/livestock Disease Investigations Team located at the USDA, National Wildlife Research Center in Fort Collins, CO, USA. Dr. Rhyan's research interests include the pathogenesis, epidemiology, detection, and management of infectious diseases at the wildlife/domestic animal/human interface, specifically, brucellosis and tuberculosis.

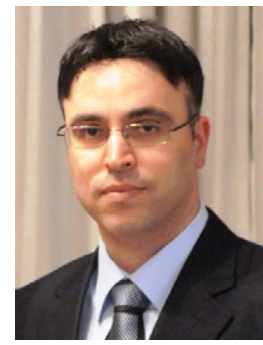

Hossam Haick is Professor in the Department of Chemical Engineering and the Russell Berrie Nanotechnology Institute and Head of the Laboratory of Nanomaterial-Based Devices at the Technion - Israel Institute of Technology. He received his Ph.D. degree in Chemical Engineering in 2002 from the Technion - Israel Institute of Technology. Haick's current research activities include nano-array devices, non-invasive disease diagnosis, volatile biomarkers, and electronic charge transport through nanomaterials. Haick is the recipient of more than 42 international honors and prizes for his research and academic achievements. 